Georgian Mathematical Journal

Volume 9 (2002), Number 3, 405-412

\title{
ON APPEL-TYPE QUADRATURE RULES
}

\author{
C. BELINGERI AND B. GERMANO
}

\begin{abstract}
The Radon technique is applied in order to recover a quadrature rule based on Appel polynomials and the so called Appel numbers. The relevant formula generalizes both the Euler-MacLaurin quadrature rule and a similar rule using Euler (instead of Bernoulli) numbers and even (instead of odd) derivatives of the given function at the endpoints of the considered interval. In the general case, the remainder term is expressed in terms of Appel numbers, and all derivatives appear. A numerical example is also included.
\end{abstract}

2000 Mathematics Subject Classification: 33C45, 33F05, 65D32.

Key words and phrases: Appel polynomials, appel numbers, EulerMacLaurin quadrature formula, differential operators.

\section{INTRODUCTION}

In the recent papers (see [3], [4], [9]) a generalization of the classical EulerMacLaurin quadrature rule has been introduced.

The new formulas are based on the Euler or, more generally, the so-called Appel numbers instead of the Bernoulli ones. By using a combination of the even derivatives computed at the endpoints of the considered interval the Eulertype quadrature rule presented in [3], [9] makes a connection of the trapezoidal rule with coefficients related to the Euler numbers.

Furthermore, in [4], an Appel-type quadrature rule considering the Appel numbers and containing all derivatives in the connection term has been introduced.

As it is well known, in [1] a general theory of quadrature rules can be found which is based on the method going back to Radon, see [2], reducing any quadrature rule known in the literature to evaluate the integral of $g(x) u(x)$, with weight function $g(x)$, to the construction of a suitable differential operator $E$, and of particular solutions of the adjoint equation $E^{*}(\varphi)=g$.

It is proved in [1] how the above-mentioned operators can be chosen in order to recover the Gaussian quadrature rules and the Euler-MacLaurin formula too.

In this paper we extend the Radon technique to the quadrature rule considered in [4].

ISSN 1072-947X / \$8.00 / C) Heldermann Verlag www.heldermann.de 


\section{Elementary Quadrature Rule in a Finite Interval}

In [1], Chapter 2, an elementary quadrature formula which is valid for real valued functions defined in a finite interval is constructed; the method can be synthetized as follows.

A quadrature formula of the type

$$
\int_{a}^{b} g(x) u(x) d x=\sum_{h=0}^{n-1} \sum_{i=1}^{m} A_{h i} u^{(h)}\left(x_{i}\right)+R(u)
$$

is constructed, where $g(x) \in L[a, b], u(x) \in A C^{n-1}[a, b]$,

$$
a=x_{0}<x_{1}<\ldots<x_{m}<x_{m+1}=b
$$

$\left(g(x)\right.$ is a weight, $u(x)$ is an argument function, $x_{1}, \ldots, x_{m}$ are nodes) under the condition that for a fixed order $n$ the linear differential operator $E$ is

$$
E=\sum_{k=0}^{n} a_{k}(x) \frac{d^{n-k}}{d x^{n-k}} .
$$

The following statement holds true:

$$
E(u)=0 \quad \Rightarrow \quad R(u)=0 .
$$

Let

$$
E_{r}(u)=\sum_{k=0}^{r} a_{k}(x) \frac{d^{r-k}}{d x^{r-k}} u, \quad r=0,1, \ldots, n-1,
$$

be the reduced operators associated with the operator $E$, and

$$
E^{*}(v)=\sum_{k=0}^{n}(-1)^{n-k} \frac{d^{n-k}}{d x^{n-k}}\left[a_{k}(x) v(x)\right] \quad\left(a_{0}(x)=1\right)
$$

the adjoint operator of $E$.

It is well known that, introducing the adjoint $E_{r}^{*}$ of the reduced operator $E_{r}$, one must assume

$$
A_{h i}=\left[E_{n-h-1}^{*}\left(\varphi_{i}(x)-\varphi_{i-1}(x)\right]_{x=x_{i}}\right.
$$

where $\varphi_{1}(x), \ldots, \varphi_{m-1}(x)$ are arbitrary solutions of the differential equations

$$
E^{*}(\varphi)=g(x)
$$

while $\varphi_{0}(x)$ and $\varphi_{m}(x)$ are integrals of the same equations (2.6) satisfying the initial conditions

$$
\varphi_{0}^{(h)}(a)=0, \quad \varphi_{m}^{(h)}(b)=0, \quad h=0,1, \ldots, n-1 .
$$

Therefore

$$
R(u)=\int_{a}^{b} \varphi(x) E(u) d x=\sum_{i=0}^{m} \int_{x_{i}}^{x_{i+1}} \varphi_{i}(x) E(u) d x,
$$

where $\varphi(x)=\varphi_{i}(x)$ with $x \in\left(x_{i}, x_{i+1}\right), i=0,1, \ldots m$. 
It is well known that the estimation of $R(u)$ can be simplified if the function $\varphi(x)$ is continuous and does not change the sign in $[a, b]$.

\section{Euler-Maclaurin Formula Using Bernoulli Polynomials}

In [1], Chapter 4, the Euler-MacLaurin formula is constructed, where the coefficients $A_{h i}$ are Bernoulli numbers. A synthesis of the method is the following.

Let us assume

$$
\begin{gathered}
a=0, \quad b=1, \quad g(x)=1, \quad x_{1}=0, \quad x_{2}=1, \\
m=2, \quad n=2 \nu+2, \quad E=\frac{d^{2 \nu+2}}{d x^{2 \nu+2}}, \quad \nu=0,1, \ldots, \\
\varphi_{1}(x)=\frac{B_{2 \nu+2}(x)-B_{2 \nu+2}}{(2 \nu+2) !}
\end{gathered}
$$

where $B_{2 \nu+2}(x)$ and $B_{2 \nu+2}$ are respectively Bernoulli polynomials and Bernoulli numbers.

The coefficients $A_{h i}$ are given by

$$
\begin{gathered}
A_{h 1}=\left\{\begin{array}{cc}
0 & (h \text { even }) \\
\frac{B_{h+1}}{(h+1) !} & (h \text { odd })
\end{array}, \quad A_{h 2}=\left\{\begin{array}{ll}
0 & (h \text { even }) \\
\frac{-B_{h+1}}{(h+1) !} & (h \text { odd })
\end{array} \quad(h=1, \ldots, 2 \nu),\right.\right. \\
A_{01}=A_{02}=\frac{1}{2} \quad \text { if } h=0, \\
A_{2 \nu+1,1}=A_{2 \nu+1,2}=0 \quad \text { if } h=2 \nu+1 .
\end{gathered}
$$

Then (2.1) becomes

$$
\int_{0}^{1} u(x) d x=\frac{1}{2}[u(0)+u(1)]+\sum_{k=1}^{\nu} \frac{B_{2 k}}{(2 k) !}\left[u^{(2 k-1)}(0)-u^{(2 k-1)}(1)\right]+R(u) ;
$$

under the hypothesis $u^{(2 \nu+2)}(x) \in C[0,1]$, equation (2.8) becomes

$$
R(u)=\int_{0}^{1} \frac{B_{2 \nu+2}(x)-B_{2 \nu+2}}{(2 \nu+2) !} u^{(2 \nu+2)}(x) d x=-\frac{B_{2 \nu+2}}{(2 \nu+2) !} u^{(2 \nu+2)}(\xi),
$$

where $0<\xi<1$.

\section{Appel Polynomials and Appel Numbers: Definition and PROPERTIES}

The Appel polynomials [10] are defined by considering the following generating function:

$$
G_{R}(x, t)=A(t) e^{x t}=\sum_{n=0}^{\infty} \frac{R_{n}(x)}{n !} t^{n},
$$


where

$$
A(t)=\sum_{k=0}^{\infty} \frac{\mathcal{R}_{k}}{k !} t^{k} \quad(A(0) \neq 0)
$$

is analytic function at $t=0$, and $\mathcal{R}_{k}:=R_{k}(0), \mathcal{R}_{0}=A(0) \neq 0$.

The numbers $\mathcal{R}_{k}$ will be called the Appel numbers associated with $A(t)$.

It easy to see that:

- if $A(t)=\frac{t}{e^{t}-1}$, then $R_{n}(t)=B_{n}(t)$

- if $A(t)=\frac{2}{e^{t}+1}$, then $R_{n}(t)=E_{n}(t)$

- if $A(t)=\alpha_{1} \ldots \alpha_{m} t^{m}\left[\left(e^{\alpha_{1} t}-1\right) \ldots\left(e^{\alpha_{m} t}-1\right)\right]^{-1}$, then $R_{n}(t)$ are Bernoulli polynomials of order $m[12]$;

- if $A(t)=2^{m}\left[\left(e^{\alpha_{1} t}+1\right) \ldots\left(e^{\alpha_{m} t}+1\right)\right]^{-1}$, then $R_{n}(t)$ are Euler polynomials of order $m[11]$.

It is suitable to introduce the coefficients $\alpha_{k}$ of the expansion:

$$
\frac{A^{\prime}(t)}{A(t)}=\sum_{n=0}^{\infty} \alpha_{n} \frac{t^{n}}{n !} .
$$

The coefficients $\alpha_{k}$ are related to the Appel numbers $\mathcal{R}_{k}$ by the relation

$$
R_{k+1}=\sum_{h=0}^{k}\left(\begin{array}{l}
k \\
h
\end{array}\right) \mathcal{R}_{h} \alpha_{k-h}
$$

The main property of Appel polynomials is expressed by the following theorem (see [4]).

Theorem 1. The only polynomials $P_{n}(x)$ satisfying the condition

$$
P_{n}^{\prime}(x)=n P_{n-1}(x), \quad n=0,1,2 \ldots,
$$

are the Appel polynomials.

A recursive computation of Appel polynomials can be obtained by using the formula

$$
R_{n+1}(x)=\left(x+\alpha_{0}\right) R_{n}(x)+\sum_{k=0}^{n-1}\left(\begin{array}{l}
n \\
k
\end{array}\right) \alpha_{n-k} R_{k}(x),
$$

$n=1,2, \ldots$, with $R_{0}(x)=\mathcal{R}_{0}=1$.

As a consequence of $(4.5)$, for the integral in $[0,1]$ of the Appel polynomials, we can write

$$
\int_{0}^{1} R_{n}(x) d x=\frac{R_{n+1}(1)-R_{n+1}(0)}{n+1}
$$




\section{Appel-Type Quadrature Formula}

In this section, starting from the Appel polynomials, we construct a quadrature rule generalizing the well known Euler-MacLaurin quadrature formula, using Appel (instead Bernoulli) numbers in the reminder term, and all (instead

only odd) order derivatives of the given function evaluated at the endpoints of the considered interval.

The Euler-MacLaurin quadrature rule can be derived as a special case, owing to the properties of Bernoulli numbers.

We can prove now our main result.

Theorem 2. Let $u(x) \in A C^{\nu+1}[0,1]$, then the quadrature rule

$$
\begin{aligned}
\int_{0}^{1} u(x) d x= & {\left[\left(1+\alpha_{0}\right) u(1)-\alpha_{0} u(0)\right] } \\
& +\sum_{k=1}^{\nu} \frac{(-1)^{k}}{(k+1) !}\left[R_{k+1}(1) u^{(k)}(1)-R_{k+1}(0) u^{(k)}(0)\right]+R(u)
\end{aligned}
$$

holds true, where the remainder $R(u)$ is expressed by

$$
R(u)=\int_{0}^{1} \frac{R_{\nu+2}(x)}{(\nu+2) !} u^{(\nu+2)}(x) d x .
$$

Proof. Let us assume

$$
\begin{gathered}
a=0, b=1, g(x)=1, m=2, x_{1}=0, x_{2}=1, n=\nu+2, \\
E=\frac{d^{\nu+2}}{d x^{\nu+2}}, \quad \nu=0,1,2, \ldots
\end{gathered}
$$

The differential equation $E^{*}(\varphi)=g(x)$ can be written as $\varphi^{(\nu+2)}(x)=1$.

It is sufficient to fix arbitrarily the integral $\varphi_{1}(x)$, for instance, we may assume

$$
\varphi_{1}(x)=\frac{R_{\nu+2}(x)}{(\nu+2) !},
$$

where $R_{\nu+2}(x)$ denotes the Appel polynomials of index $\nu+2$.

By using the hypothesis $u(x) \in A C^{\nu+2}[0,1]$, equations (2.1), (2.8) become respectively

$$
\begin{aligned}
\int_{0}^{1} u(x) d x & =\sum_{h=0}^{\nu+2}\left[A_{h 1} u^{(h)}(0)+A_{h 2} u^{(h)}(1)\right]+R(u), \\
R(u) & =\int_{0}^{1} \frac{R_{\nu+2}(x)}{(\nu+2) !} u^{(\nu+2)}(x) d x .
\end{aligned}
$$

Now we have only to compute the coefficients $A_{h 1}, A_{h 2}$ using (2.5). 
We find

$$
A_{h 1}=\left[E_{\nu-1-h}^{*}\left(\varphi_{1}\right)\right]_{x=0}=(-1)^{\nu+1-h} \varphi_{1}^{(\nu+1-h)}(0), \quad h=0,1, \ldots, \nu
$$

so that

$$
\begin{gathered}
A_{h 1}=(-1)^{h+1} \frac{R_{h+1}(0)}{(h+1) !} \\
A_{h 2}=\left[E_{\nu+1-h}^{*}\left(-\varphi_{1}\right)\right]_{x=1}=-(-1)^{\nu+1-h} \varphi_{1}^{(\nu+1-h)}(1), \quad h=0,1, \ldots, \nu,
\end{gathered}
$$

so that

$$
A_{h 2}=(-1)^{h} \frac{R_{h+1}(1)}{(h+1) !}
$$

and therefore, recalling (4.6),

$$
A_{01}=-\alpha_{0}, \quad A_{02}=1+\alpha_{0} .
$$

Hence, recalling the hypothesis $u^{(\nu+2)}(x) \in C[0,1]$, equations (5.2) and (5.3) become respectively

$$
\begin{aligned}
\int_{0}^{1} u(x) d x= & {\left[\left(1+\alpha_{0}\right) u(1)-\alpha_{0} u(0)\right]+\sum_{k=1}^{\nu} \frac{(-1)^{k}}{(k+1) !}\left[R_{k+1}(1) u^{(k)}(1)\right.} \\
& \left.-R_{k+1}(0) u^{(k)}(0)\right]+R(u), \\
R(u)= & \int_{0}^{1} \frac{R_{\nu+2}(x)}{(\nu+2) !} u^{(\nu+2)}(x) d x=u^{(\nu+2)}(\xi) \int_{0}^{1} \frac{R_{\nu+2}(x)}{(\nu+2) !} d x \\
= & u^{(\nu+2)}(\xi) \frac{R_{\nu+3}(1)-R_{\nu+3}(0)}{(\nu+3) !}, \quad 0<\xi<1, \quad \nu=0,1,2 \ldots
\end{aligned}
$$

\section{A Numerical Example}

Note that the Appel quadrature rule depends on a choice of the function $A(t)$ so that infinitely many formulas can be deduced. In the following we will assume $A(t)=e^{e^{-\frac{t}{N}}-1}$, where $N \geq 1$ is an integer. In this case we find

$$
\alpha_{k}=\frac{(-1)^{k+1}}{N^{k+1}}
$$

so that $R_{k+1}$ are expressed by

$$
\mathcal{R}_{k+1}=-\frac{1}{N} \sum_{h=0}^{k}\left(\begin{array}{l}
k \\
h
\end{array}\right) \frac{(-1)^{k-h}}{N^{k-h}} \mathcal{R}_{h}
$$


The first Appel polynomials and the corresponding Appel numbers are given by

$$
\begin{aligned}
& R_{0}(x)=1 \\
& R_{1}(x)=x-\frac{1}{N}, \\
& R_{2}(x)=x^{2}-\frac{2}{N} x+\frac{2}{N^{2}}, \\
& R_{3}(x)=x^{3}-\frac{3}{N} x^{2}+\frac{6}{N^{2}} x-\frac{5}{N^{3}}, \\
& R_{4}(x)=x^{4}-\frac{4}{N} x^{3}+\frac{12}{N^{2}} x^{2}-\frac{20}{N^{3}} x+\frac{15}{N^{4}}, \\
& R_{5}(x)=x^{5}-\frac{5}{N} x^{4}+\frac{20}{N^{2}} x^{3}-\frac{50}{N^{3}} x^{2}+\frac{75}{N^{4}} x-\frac{52}{N^{5}}, \\
& R_{6}(x)=x^{6}-\frac{6}{N} x^{5}+\frac{30}{N^{2}} x^{4}-\frac{100}{N^{3}} x^{3}+\frac{225}{N^{4}} x^{2}-\frac{312}{N^{5}} x+\frac{203}{N^{6}}, \\
& R_{7}(x)=x^{7}-\frac{7}{N} x^{6}+\frac{42}{N^{2}} x^{5}-\frac{175}{N^{3}} x^{4}+\frac{525}{N^{4}} x^{3}-\frac{1092}{N^{5}} x^{2}+\frac{1391}{N^{6}} x-\frac{877}{N^{7}} .
\end{aligned}
$$

Let us apply the quadrature formula (5.4) with $\nu=6$, assuming the above choice for $A(t)$ and $N=161098$, in order to integrate the function

$$
u(x)=\frac{\sin x}{x} .
$$

Then we can write

$$
I=\int_{0}^{1} \frac{\sin x}{x} d x=u(1)+\sum_{k=1}^{6} \frac{(-1)^{k}}{(k+1) !}\left[R_{k+1}(1) u^{(k)}(1)-R_{k+1}(0) u^{(k)}(0)\right]+R(u),
$$

and consequently, by using the computer algebra program Derive V.4.07, we find

$$
I=0.946083070365
$$

with an error term less than $10^{-11}$, while the computation of the same integral, using the Taylor expansion with seven terms, gives a worse result, namely we found the approximation 0.946083070354 with an error less than $10^{-10}$.

It is worth noting that the choice of the integer $N$ depends on the accuracy we want to obtain: increasing its value we can obtain better results.

Remark. It is worth supposing that an interesting open problem is connected with the possibility to choice in a suitable way the function $A(t)$ in order to have the smallest possible error depending on the class of functions we have to integrate. 


\section{REFERENCES}

1. A. Ghizzetti and A. Ossicini, Quadrature formulae. Academic Press, New York, 1970.

2. J. RAdon, Restausdrücke bei Interpolations- und Quadraturformeln durch bestimmte Integrale. Monatsh. Math. Phys. 42(1935), 389-396.

3. G. Bretti and P. E. RicCi, Euler polynomials and the related quadrature rule. Georgian Math. J. 8(2001), No. 3, 447-453.

4. G. Bretti, M. X. He, and P. E. Ricci, On quadrature rules associated with Appel polynomials. (Submitted)

5. J. Stoer, Introduzione all'Analisi Numerica. Zanichelli, Bologna, 1972.

6. L. Euler, Instituziones calculi differentialis. Opera Omnia, Series prima: opera mathematica 10, G. Kowalewski Ed., Teubner, 1980.

7. I. S. Gradshteyn and I. M. Ryzhik, Table of integrals, series and products. Academie Press, New York, 1980.

8. M. Abramowitz and I. A. Stegun, Handbook of mathematical functions. Dover, New York, 1965.

9. C. Belingeri and G. Bretti, An Euler-type quadrature rule derived by using Radon's method. (Submitted)

10. P. APPEL and J. KAMPÉ DE FÉRIET, Fonctions hypergéometriques et hypersphériques. Polinômes d'Hermite. Gauthier-Villars, Paris, 1926.

11. A. Erdélyi, W. Magnus, F. Oberhettinger, and F. G. Tricomi, Higher transcendental functions. Vols. I, II. Based, in part, on notes left by Harry Bateman. McGraw-Hill Book Company, Inc., New York-Toronto-London, 1953.

(Received 28.09.2001)

Authors' address:

Dipartimento di Metodi e Modelli Matematici

per le Scienze Applicate

Università degli Studi di Roma "La Sapienza"

Via A. Scarpa 14, 00161 Roma

Italy

E-mail: belingeri@dmmm.uniroma1.it

germano@dmmm.uniroma1.it 